\title{
Insular epilepsy, an under-recognized seizure semiology
}

\section{A review for general neurologist}

Bandar N. Aljafen, MD.

\begin{abstract}

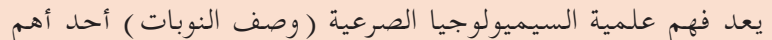

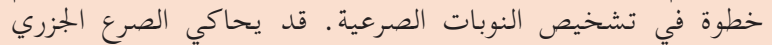

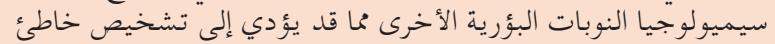

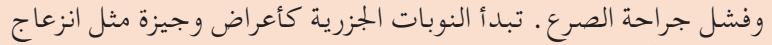

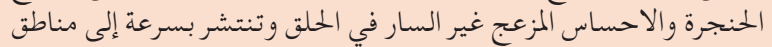

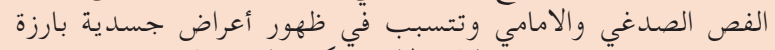

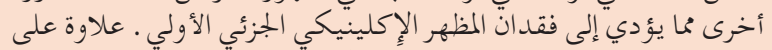

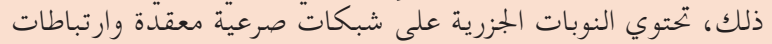

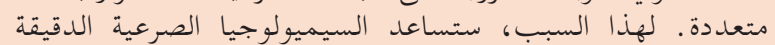

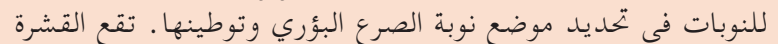

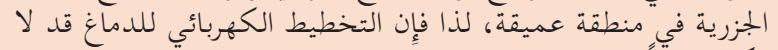

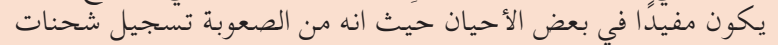

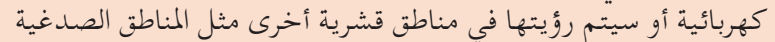

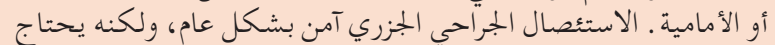

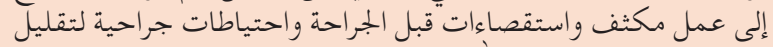
احتمالية الوفيات لا قدر الله ولهي
\end{abstract}

Understanding seizure semiology is one of the most important and crucial steps in diagnosing a seizure disorder. Insular epilepsy may mimic other focal seizure semiologies, leading to misdiagnosis and failed epilepsy surgery. Insular seizures may begin as brief ictal symptoms, such as laryngeal discomfort and unpleasant throat sensations, and spread rapidly to the temporal or frontal regions, causing prominent ictal symptoms different to the initial insular ictal manifestation. Moreover, insular seizures are associated with complex epileptogenic networks and multiple connections. For this reason, accurate seizure semiology helps to lateralize and localize the seizure onset. The insular cortex is deep, and thus scalp electroencephalography is not always beneficial as the epileptic discharges will not be easily recorded, or they will be seen over other cortical regions like the temporal or frontal areas. Insular surgical resection is generally safe, but it requires extensive presurgical workup and surgical precautions in order to minimize mortality.

Neurosciences 2020; Vol. 25 (4): 262-268 doi: $10.17712 / n s j .2020 .4 .20200063$

From the College of Medicine, King Saud University, Riyadh, Kingdom of Saudi Arabia

Received 27th April 2020. Accepted 20th June 2020.

Address correspondence and reprint request to: Dr. Bandar N. Aljafen, College of Medicine, King Saud University, Riyadh, Kingdom of Saudi Arabia.E-mail: baljafen@ksu.edu.sa

ORCID ID: https://orcid.org/0000-0001-8845-4020

I nsular seizures are a great mimicker of temporal, frontal, and parietal seizure semiology. ${ }^{1-3}$ Insular epilepsy is considered to be one of the causes of failure of temporal lobe epilepsy surgery. ${ }^{4,5}$ This concept was proposed initially by Penfield in the 1950s, and then by Isnard et al., who perform intracranial monitoring for epileptic patients with atypical temporal lobe epilepsy; ${ }^{4-}$ ${ }^{6}$ they found that patients with an insular seizure onset had a unique ictal seizure semiology, including laryngeal constriction and unpleasant sensory symptoms in full consciousness. ${ }^{6}$ Subsequently, the interest in insular ictal semiology has increased, leading to the reporting of more insular ictal symptoms such as hypersalivation, dysarthria, and focal motor manifestations. ${ }^{7}$

The aim of this review is to understand insular seizure semiology, electroencephalogram (EEG) findings, surgical treatment, and outcomes in insular lobe epilepsy, in order to increase recognition of this rare seizure type. To facilitate this, electronic databases were systematically searched, including MEDLINE (PubMed and Ovid), EMBASE, and Google Scholar, to identify all published studies on insular epilepsy and semiology. Studies with a good design were included in

Disclosure. The authors declare no conflicting interests, support or funding from any drug company. 
our review. The reference lists of all potential studies were reviewed to identify additional relevant manuscripts.

Insular lobe anatomy. The insula was first described by Johann Christian Reil in 1809; Henry Gray called it "Island of Reil" in Gray's Anatomy." More than 100 years ago, Clark was one of the first authors that discussed and described the position of the insular lobe, and called it the fifth lobe of the brain. ${ }^{8}$ The insula is a triangular folded part of the cerebral cortex, lying deep underneath the lateral cerebral fissure in both hemispheres. It is completely covered by the temporal, parietal, and frontal opercula. Despite its deep location within both hemispheres, it is considered one of the cortical components, and can be exposed by separating the upper and lower parts of the lateral fissure (Figure 1). ${ }^{9}$ A central insular sulcus divides it into anterior and posterior parts, consisting of three short gyri anteriorly and 2 long gyri posteriorly (Figure 1). ${ }^{10}$ Based on cytoarchitectonical cellular divisions, the insula can also be subdivided into 3 zones: anterior agranular, precentral dysgranular, and posterior granular. ${ }^{11}$

Embryologically, the insular cortex (lobus insularis) is one of the earliest parts of the human cortex to develop, in the sixth gestational week. ${ }^{12}$ Afif et $\mathrm{al}^{13}$ divided the morphological stages of insular gyral and sulcal development into 5 stages, corresponding to the gestational age, starting from the appearance of the first sulcus at 13-17 weeks of intrauterine fetal life, to the closure of the sylvian fissure at 27-28 gestational weeks. The insula receives its blood supply mainly from the M2 segment of the middle cerebral artery (MCA). In addition to M2, the insula is also supplied by the M1 and M3 segments to some extent, and the accessory MCA from the anterior cerebral artery. ${ }^{14,15}$ The venous drainage of the insula is mainly to the deep middle cerebral vein and the superficial sylvian vein. ${ }^{15}$
Function. The physiological functions of the insular lobe have been extensively researched. It has been found to have special functions related to its afferent and efferent projections to various cortical and deep structures. The afferent and efferent connections of the insula are summarized in Figure 2. ${ }^{16}$ Most of the insular functions were identified by cortical stimulation and, to a lesser extent, by functional magnetic resonance imaging (fMRI), especially with regards to language function. Different studies have suggested that the insular cortex plays a major role in somatosensory, viscerosensory, cognitive, behavioral, and autonomic symptoms (bradycardia, nausea, vomiting, piloerection), as well as language and motor functions. ${ }^{17-20}$ More specifically, the anterior insula is responsible for emotional experiences and self-awareness, while the posterior insula is important for different pain modalities (pain, touch and temperature) as it receives a direct input from the thalamus. ${ }^{12,21,22}$

Symptoms of insular cortex pathology. Pathology of the insular cortex may generate a variety of symptoms, depending on which part of the insular cortex is affected. Seizures with specific characteristics, including unpleasant somatosensory feelings, laryngeal constriction, hypersalivation, and dysphonic or dysarthric symptoms, are the most important manifestations of insular pathology. ${ }^{6}$ Seizures originating from the anterior insula may be asymptomatic at onset but may propagate and spread rapidly, leading to hypermotor symptoms, while posterior insular seizures will cause contralateral somatosensory symptoms. ${ }^{23}$

Goodkind et $\mathrm{al}^{24}$ recently published a meta-analysis on functional and other structural neuroimaging studies that showed a variety of insular abnormalities were associated with neuropsychiatric disorders such as depression, anxiety, addiction, autism, and

Table 1 - Insular versus temporal versus frontal ictal clinical features.

\begin{tabular}{lccc}
\hline Clinical features & Insular & Temporal & Frontal \\
\hline Painful somatosensory & Common & No & No \\
Throat sensation & Common & Rare & No \\
Laryngeal constriction & Common & Rare & No \\
Hyper-motor & Yes & No & common \\
Orofacial sensation & Common & Rare & No \\
Viscerovegetative signs, including & Common & Rare & No \\
hypersalivation, nausea, facial blush, & & & Yes (dominant side) \\
sweaty hands and urge to urinate & Yes (dominant side) & Yes (dominant side) & No \\
$\begin{array}{l}\text { Speech arrest/dysarthria } \\
\text { Epigastric rising sensation }\end{array}$ & Rare & Yes & Brief \\
$\begin{array}{l}\text { Altered awareness and loss of } \\
\text { consciousness }\end{array}$ & No & Yes & \\
\hline
\end{tabular}



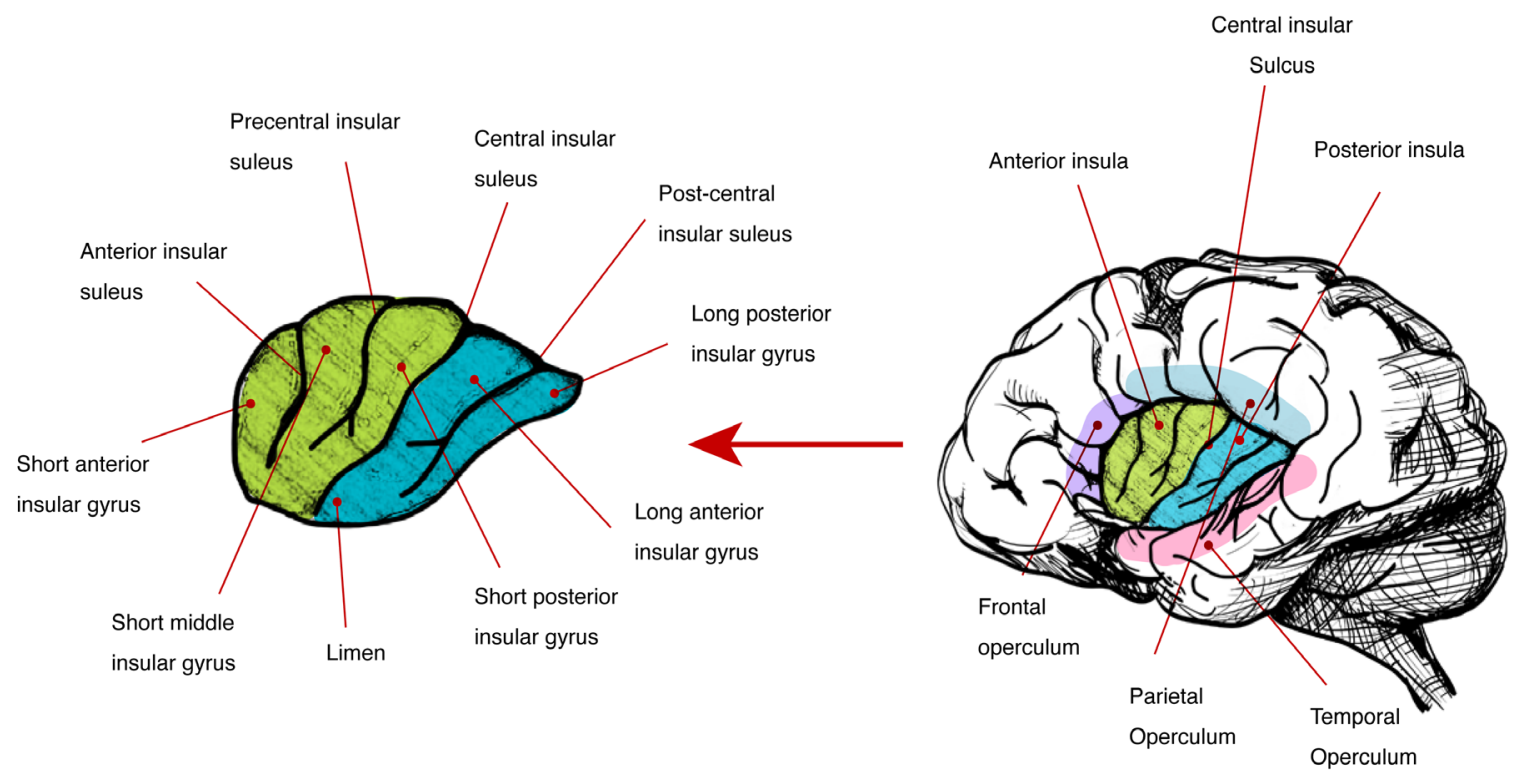

Figure 1 - Insular cortex anatomy

schizophrenia. ${ }^{24-26}$ Insular lesions may also cause various cognitive, autonomic, and language dysfunctions. ${ }^{17,18,27}$ Despite the role of the insula in aphasia and language dysfunction being unclear, it has been found that language dysfunction is associated with dominant insular lesions. ${ }^{22}$ Different pathological lesions can cause insular symptoms, Chevrier et al. reviewed the structural abnormalities in 48 patients with insular/ peri-insular cortex epilepsy and found neoplastic lesions in $27 \%$ of the patients, cortical malformations in $21 \%$, vascular malformations in 19\%, and encephalomalacia and atrophy in $17 \% .{ }^{28}$ Moreover, other studies have reported etiologies for insular symptoms to include insular lesions such as low-grade brain tumors, vascular abnormalities including cavernomas, focal cortical dysplasia, and gliosis, the latter of which could be posttraumatic or related to an old brain insult. ${ }^{29,30}$

Seizure semiology in insular epilepsy. Isnard et $\mathrm{a}^{6}$ using intracranial monitoring with depth electrodes, were the first authors to analyze a special ictal manifestation in patients with insular epilepsy. They found that all patients had a preserved conscious level at onset. The majority of patients reported laryngeal discomfort and unpleasant throat sensations at seizure onset, and these symptoms varied in intensity, ranging from mild intensity to a strong sensation of strangulation. Insular seizure could be associated with retrosternal pain, abdominal heaviness, or shortness of breath. In addition to that, some patients reported speech abnormalities such as dysphonia or dysarthria, which progressed to a complete inability to talk for a few seconds to a couple of minutes, with associated lateralized somatosensory or somatomotor focal signs. ${ }^{1,6}$

Mazzola et $\mathrm{al}^{31}$ reviewed stereotactic stimulation of the insular cortex using stereo-electro-encephalography (SEEG), aiming to identify the seizure semiology in 222 patients admitted to an epilepsy monitoring unit for presurgical evaluation. The patients presented with either atypical temporal or insular lobe epilepsy. Ictal symptoms evoked by insular stimulation were multiple and consistent with perisylvian ictal symptoms such as somatosensory symptoms, including pain or laryngeal spasm, in addition to auditory, speech, vestibular, or olfacto-gustatory manifestations. ${ }^{31}$ Somatosensory ictal symptoms have been shown to manifest as paresthesia followed by pain and thermal sensations. ${ }^{32,33}$ Ostrowsky et $\mathrm{al}^{34}$ using insular stimulation, were the first investigators to describe pain as an insular cortex ictal symptom; the pain sensation was described as an electrical shock, burning, and painful pins and needles. ${ }^{34}$

Visceral ictal symptoms have been described as a constrictive sensation and discomfort in the laryngeal, retrosternal, or abdominal regions. In addition to these ictal visceral symptoms, patients with insular epilepsy may experience salivation as well as facial blush and, less commonly, the urge to urinate and sweaty hands. ${ }^{33}$

The insular cortex is part of a complex epileptogenic network with multiple connections to different cortical and subcortical regions (Figure 2). ${ }^{35}$ For this reason, insular epilepsy is considered to be a 'great mimicker', 


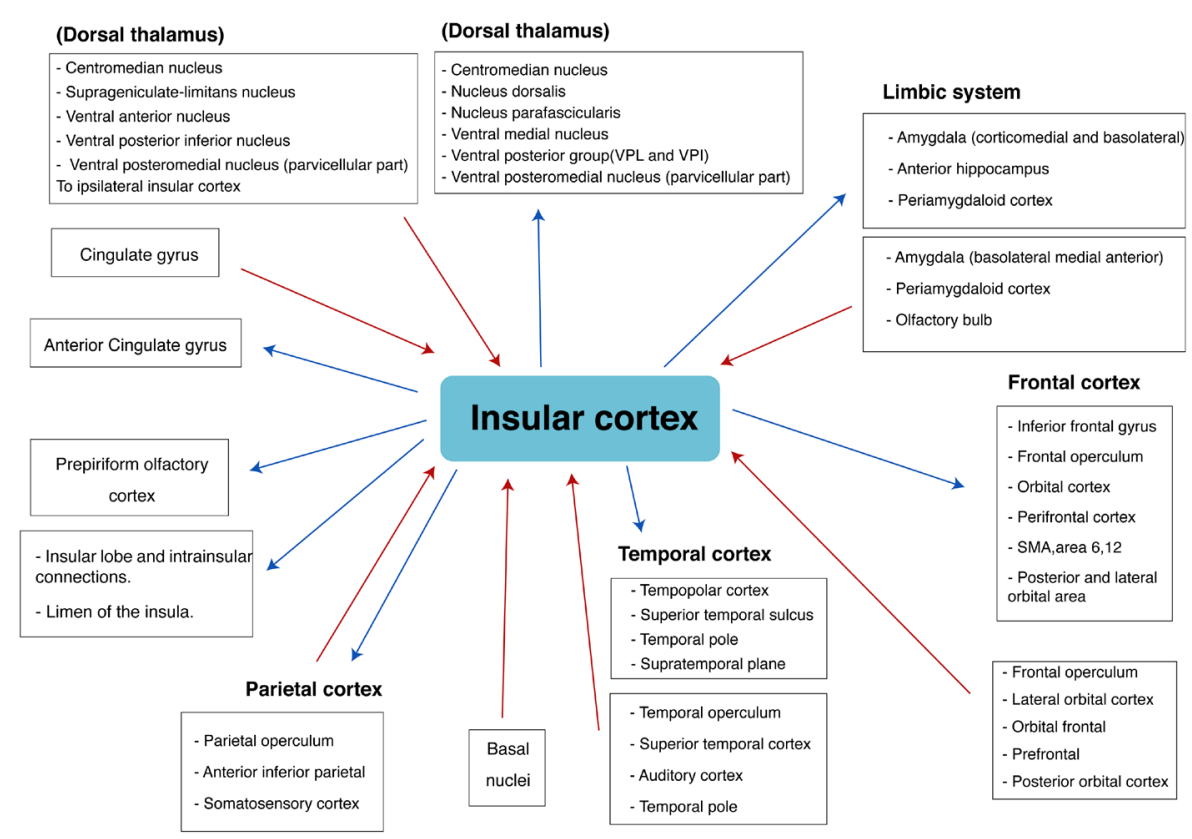

Figure 2 - The afferent and efferent connection of insular cortex, this figure is adopted with permission and modification from Augustine et al 1996 (16)

because in addition to the perisylvian manifestations, it may present with temporal or frontal lobe ictal symptoms (Table 1). Some of these temporal ictal symptoms are oral or manual automatism, and focal dyscognitive manifestations. On the other hand, frontal ictal symptoms identified in insular seizures include hypermotor or behavioral symptoms. ${ }^{2,36-38}$

Ryvlin et $\mathrm{al}^{2}$ using intracranial EEG monitoring, evaluated 3 patients with drug-resistant hypermotor seizures diagnosed either as non-lesional or autosomal dominant nocturnal frontal lobe epilepsy (ADNFLE). They found that the ictal onset in all three patients originated in the anterosuperior part of the insular cortex, indicating that the insula may play a major role in nocturnal hypermotor seizures. ${ }^{2}$

Dylgieri et $\mathrm{al}^{39}$ described insular ictal semiology in a pediatric age group which was characterized by autonomic manifestations, spasms, and myoclonic jerks with asymmetric atonic and hypermotor seizures. Freri et $\mathrm{al}^{40}$ retrospectively reviewed 16 pediatric patients with drug-resistant epilepsy who were suspected to have perisylvian and insular seizures, and were admitted to the epilepsy monitoring unit for presurgical evaluation. In all the patients, seizures were captured from the insular cortex. The semiology included perioral focal motor seizures associated with auditory hallucinations, unpleasant paresthesias, and epigastric rising sensations in $75 \%$ of the patients. Autonomic manifestations were noted in $50 \%$ of the patients.
Scalp EEG findings in insular epilepsy. EEG plays an important and crucial role in the diagnosis of epilepsy and seizure localization. The sensitivity of EEG in detecting epileptiform discharges depends upon multiple factors including seizure frequency, repeating the EEG, use of a prolonged recording, and performing the EEG within 48 hours of the seizure attack. All these factors will increase the detection of epileptiform discharges in patients with epilepsy. ${ }^{41}$ Given that the insula lies deep in both hemispheres and is totally covered by the temporal, parietal, and frontal opercula, it is difficult to detect interictal or ictal insular seizure activities using scalp EEG. ${ }^{17}$ The insular interictal epileptiform discharges (IEDs) may be seen over the frontotemporal, frontopolar, or mid-temporal regions. ${ }^{3,42}$

Levy et $\mathrm{al}^{42}$ reported the scalp and intracranial EEG findings in nine patients with definite operculoinsular epilepsy, confirmed by intracranial EEG, and good seizure outcomes after surgical resection or radiosurgery. The analysis of the scalp EEG in all patients revealed 14 types of IEDs. The frequent IEDs were electronegative discharges over either the frontopolar or frontotemporal and mid-temporal regions. The other types of IEDs were infrequent central or temporal discharges. None of the patients had parietal or occipital interictal discharges. The morphology of the IEDs were either spikes, bursts of low amplitude spikes, or brief low voltage fast activities (LVFA). Ten ictal scalp EEG seizures were 
captured using video EEG recording. Four out of these ten seizures showed no clear ictal scalp EEG changes at onset; the other 6 seizures showed a clear lateralized ictal rhythm. The ictal rhythm patterns include rhythmic spikes and waves, LVFA, rhythmic alpha activity, and rhythmic delta activity. ${ }^{42}$

Intracranial EEG findings in insular epilepsy. Intracranial EEG (icEEG) is the gold standard and most useful diagnostic study for seizure localization in drugresistant epilepsy with atypical frontal, orbitofrontal, or temporal lobe seizures that may have originated from the insular cortex. ${ }^{37,42}$ Isnard et al. were the first investigators who describe icEEG using depth electrodes within the insula to record insular lobe seizures; it was considered a dangerous and unsafe procedure. ${ }^{1}$ After that study, several reports of icEEG monitoring of the insular cortex were published, indicating that the procedure was useful in seizure localization and in obtaining more information about insular function, using cortical mapping and electrical stimulation. ${ }^{3,6,39,42}$

Once insular epilepsy is suspected, and there is a clear hypothesis that the seizure onset may have originated from the insula, then an icEEG is indicated prior to performing epilepsy surgery, especially in cases of nonlesional epilepsy. ${ }^{37,43,44}$ There is a different approach for icEEG implantation in the insular cortex using either depth or subdural electrodes, or both. ${ }^{44}$ Despite the insular cortex being a highly vascular region of the brain, icEEG implantation is still a safe procedure if it is carefully applied. The choice to implant depth or subdural electrodes or both should be individualized to the specific patient. ${ }^{43-46}$

Levy et $\mathrm{al}^{42}$ found different icEEG seizure onset patterns in patients with definite operculoinsular epilepsy. The most common seizure onset pattern seen on icEEG is low-voltage fast activity followed by rhythmic spike waves, delta brushes, and sharp waves. Levy et $\mathrm{al}^{42}$ also found that the ictal rhythm may be seen simultaneously on the extra-insular electrodes at seizure onset in some patients. In their study, all seizures propagated and spread outside the insular cortex, which may explain the variable clinical manifestations.

Surgical outcomes in insular epilepsy. Penfield et al. and Guillaume et al. in the late 1940s were the first to attempt insular resection for intractable epilepsy. ${ }^{4,5,47}$ Unfortunately, the idea of insular surgery was initially neglected and ignored, due to a high mortality and poor surgical outcomes. ${ }^{48}$

Subsequently, a series of successful lesional insular epilepsy surgeries were published. ${ }^{30,49,50}$ Yasargil et al. reported the outcomes of 177 patients with limbic and paralimbic tumors, including 80 patients with insular tumors; they described good outcomes and no operative mortality, and more than $84 \%$ seizure freedom post-tumor resection. ${ }^{49}$ von Lehe et $\mathrm{al}^{51}$ reported a series of 26 insular lesionectomies which resulted in a seizure outcome satisfaction rate of up to $79.2 \%$ postlesionectomy.

The decision regarding surgical resection in nonlesional insular epilepsy cases is challenging and requires adequate presurgical workup for accurate seizure localization. Malak et $\mathrm{al}^{52}$ described nine patients who underwent insular surgery due to refractory epilepsy; among them, seven patients had non-lesional insular epilepsy with an Engel Class IA outcome in six patients and an Engel Class III outcome in one patient. ${ }^{52}$ Alomar et $\mathrm{al}^{46}$ also reported a series of successful non-lesional insular epilepsy surgeries, with $33.3 \%$ of patients associated with an Engel Class I outcome, $40 \%$ with an Engel Class II outcome, 20\% with an Engel Class III outcome, and 6.66\% with an Engel Class IV outcome. Insular resection is generally safe, with mild and transient complications, except for some permanent motor deficits that can result from open resection of the caudal dorsal insula and adjacent parietal operculum. ${ }^{33,46}$ To avoid this possible motor complication, magnetic resonance imaging-guided stereotactic laser ablation to the dorsal caudal insula is an alternative and recommended safe procedure. ${ }^{33}$ In summary, the recent literature supports surgical interventions for lesional and non-lesional insular epilepsy, and should be considered in any drugresistant insular epilepsy cases.

In conclusion, insular lobe seizures are an underrecognized seizure type that require special attention and careful history taking. Understanding the insular seizure semiology is a crucial and essential step in obtaining an accurate diagnosis. Insular semiological features should be correlated with the MRI and video-EEG changes, which will determine the subsequent medical and surgical treatment options aimed at obtaining satisfactory seizure outcomes.

Acknowledgment. The author would like to acknowledges the efforts of the artist Dalia M. Almosa for her help in the creation of the anatomical pictures and the figure designs. The author also extends gratitude for Dr. Mohammed Abuelnor, PhD, for his comments on the anatomical section. Finally, the author would like to thank Editage (www.editage.com) for English language editing.

\section{References}

1. Isnard J, Guénot M, Ostrowsky K, Sindou M, Mauguière F. The role of the insular cortex in temporal lobe epilepsy. Ann Neurol 2000; 48: 614-623.

2. Ryvlin P, Minotti L, Demarquay G, Hirsch E, Arzimanoglou A, Hoffman D, et al. Nocturnal hypermotor seizures, suggesting frontal lobe epilepsy, can originate in the insula. Epilepsia 2006; 47: 755-765. 
3. Proserpio P, Cossu M, Francione S, Tassi L, Mai R, Didato G, et al. Insular-opercular seizures manifesting with sleep-related paroxysmal motor behaviors: a stereo-EEG study. Epilepsia 2011; 52: 1781-1791.

4. Penfield W, Jasper H. Epilepsy and the functional anatomy of the human brain. Boston (MA): Little Brown \& Co.; 1954.

5. Penfield W, Faulk M. The insula. Brain 1955; 78: 445-470.

6. Isnard J, Guénot M, Sindou M, Mauguière F. Clinical manifestations of insular lobe seizures: a stereoelectroencephalographic study. Epilepsia 2004; 45: 1079-1090.

7. Binder DK, Schaller K, Clusmann H. The seminal contributions of Johann-Christian Reil to anatomy, physiology, and psychiatry. Neurosurgery 2007; 61: 1091-1096.

8. Clark TE. The comparative anatomy of the insula. J Comp Neurol 1896; 6: 59-100.

9. Waxman SG. Clinical neuroanatomy, 26th edition. New York (NY): McGraw Hill Professional; 2010.

10. Türe U, Yaşargil DC, Al-Mefty O, Yaşargil MG. Topographic anatomy of the insular region. J Neurosurg 1999; 90: 720-733.

11. Morel A, Gallay MN, Baechler A, Wyss M, Gallay DS. The human insula: Architectonic organization and postmortem MRI registration. Neuroscience 2013; 23: 117-135.

12. Kalani MY, Kalani MA, Gwinn R, Keogh B, Tse VC. Embryological development of the human insula and its implications for the spread and resection of insular gliomas. Neurosurg Focus 2009; 27: E2.

13. Afif A, Bouvier R, Buenerd A, Trouillas J, Mertens P. Development of the human fetal insular cortex: Study of the gyration from 13 to 28 gestational weeks. Brain Struct Funct 2007; 212: 335.

14. Türe U, Yaşargil MG, Al-Mefty O, Yaşargil DC. Arteries of the insula. J Neurosurg 2000; 92: 676-687.

15. Tanriover N, Rhoton AL Jr, Kawashima M, Ulm AJ, Yasuda A. Microsurgical anatomy of the insula and the sylvian fissure. $J$ Neurosurg 2004; 100: 891-922.

16. Augustine JR. Circuitry and functional aspects of the insular lobe in primates including humans. Brain Res Rev 1996; 22: 229-244.

17. Laoprasert P, Ojemann JG, Handler MH. Insular epilepsy surgery. Epilepsia 2017; 58: 35-45.

18. Meyer S, Strittmatter M, Fischer C, Georg T, Schmitz B. Lateralization in autonomic dysfunction in ischemic stroke involving the insular cortex. Neuroreport 2004; 15: 357-361.

19. Liu Z, Xu C, Xu Y, Wang Y, Zhao B, Lv Y, et al. Decreased regional homogeneity in insula and cerebellum: A resting-state fMRI study in patients with major depression and subjects at high risk for major depression. Psychiat Res 2010; 182: 211-215.

20. Chang LJ, Yarkoni T, Khaw MW, Sanfey AG. Decoding the Role of the Insula in Human Cognition: Functional Parcellation and Large-Scale Reverse Inference. Cereb Cortex 2013; 23: 739-749.

21. Damasio AR, Grabowski TJ, Bechara A, Damasio H, Ponto LL, Parvizi J, et al. Subcortical and cortical brain activity during the feeling of self-generated emotions. Nat Neurosci 2000; 3: 1049-1056.

22. Gasquoine PG. Contributions of the insula to cognition and emotion. Neuropsychol Rev 2014; 24: 77-87.

23. Wynford-Thomas R, Powell R. Navigating the Island of Reil: how to understand the insular cortex. Pract Neurol 2017; 17: $122-126$
24. Goodkind M, Eickhoff SB, Oathes DJ, Jiang Y, Chang A, JonesHagata LB, et al. Identification of a common neurobiological substrate for mental illness. JAMA Psychiatry 2015; 72: 305-315.

25. Stratmann M, Konrad C, Kugel H, Krug A, Schöning S, Ohrmann $\mathrm{P}$, et al. Insular and hippocampal gray matter volume reductions in patients with major depressive disorder. PLoS One 2014; 9: e102692.

26. Wylie KP, Tregellas JR. The role of the insula in schizophrenia. Schizophr Res 2010; 123: 93-104.

27. Afif A, Minotti L, Kahane P, Hoffmann D. Middle short gyrus of the insula implicated in speech production: intracerebral electric stimulation of patients with epilepsy. Epilepsia 2010; 51: 206-213.

28. Chevrier MC, Bard C, Guilbert F, Nguyen DK. Structural abnormalities in patients with insular/peri-insular epilepsy: spectrum, frequency, and pharmacoresistance. Am J Neuroradiol 2013; 34: 2152-2156.

29. Roper SN, Lévesque MF, Sutherling WW, Engel J Jr. Surgical treatment of partial epilepsy arising from the insular cortex: report of two cases. J Neurosurg 1993; 79: 266-269.

30. Duffau H, Capelle L, Lopes M, Bitar A, Sichez JP, van Effenterre R. Medically intractable epilepsy from insular low-grade gliomas: improvement after an extended lesionectomy. Acta Neurochir 2002; 144: 563-573.

31. Mazzola L, Mauguiere F, Isnard J. Electrical stimulations of the human insula: their contribution to the ictal semiology of insular seizures. J Clin Neurophysiol 2017; 34: 307-314.

32. Pugnaghi M, Meletti S, Castana L, Francione S, Nobili L, Mai $\mathrm{R}$, et al. Features of somatosensory manifestations induced by intracranial electrical stimulations of the human insula. Clin Neurophysiol 2011; 122: 2049-2058.

33. Jobst BC, Gonzalez-Martinez J, Isnard J, Kahane P, Lacuey N, Lahtoo SD, et al. The Insula and Its Epilepsies, Epilepsy Currents 2019; 19: 11-21.

34. Ostrowsky K, Magnin M, Ryvlin P, Isnard J, Guenot M, Mauguiere F. Representation of pain and somatic sensation in the human insula: a study of responses to direct electrical cortical stimulation. Cereb Cortex 2002; 12: 376-385.

35. Namkung H, Kim SH, Sawa A. The insula: an underestimated brain area in clinical neuroscience, psychiatry, and neurology. Trends Neurosci 2017; 40: 200-207.

36. Blauwblomme T, Kahane P, Minotti L, Grouiller F, Krainik A, Vercueil L, et al. Multimodal imaging reveals the role of gamma activity in eating-reflex seizures. J Neurol Neurosurg Psychiatry 2011; 82: 1171-1173.

37. Kriegel MF, Roberts DW, Jobst BC. Orbitofrontal and insular epilepsy. J Clin Neurophysiol 2012; 29: 385-391.

38. Fei P, Soucy JP, Obaid S, Boucher O, Bouthillier A, Nguyen DK. The value of regional cerebral blood flow SPECT and FDG PET in operculoinsular epilepsy. Clin Nucl Med 2018; 43: e67-e73.

39. Dylgjeri S, Taussig D, Chipaux M, Lebas A, Fohlen M, Bulteau C, et al. Insular and insuloopercular epilepsy in childhood: an SEEG study. Seizure 2014; 23: 300-308.

40. Freri E, Matricardi S, Gozzo F, Cossu M, Granata T, Tassi L. Perisylvian, including insular, childhood epilepsy: presurgical workup and surgical outcome. Epilepsia 2017; 58: 1360-1369.

41. Sundaram M, Hogan T, Hiscock M, Pillay N. Factors affecting interictal spike discharges in adults with epilepsy. Electroencephalogr Clin Neurophysiol 1990; 75: 358-360. 
42. Levy A, Yen Tran TP, Boucher O, Bouthillier A, Nguyen DK. Operculo-insular epilepsy: scalp and intracranial electroencephalographic findings. J Clin Neurophysiol 2017; 34: 438-447.

43. Afif A, Chabardes S, Minotti L, Kahane P, Hoffmann D. Safety and usefulness of insular depth electrodes implanted via an oblique approach in patients with epilepsy. Neurosurgery 2008; 62: ONS471-ONS480.

44. Surbeck W, Bouthillier A, Weil AG, Crevier L, Carmant L, Lortie A, et al. The combination of subdural and depth electrodes for intracranial EEG investigation of suspected insular (perisylvian) epilepsy. Epilepsia 2011; 52: 458-466.

45. Nguyen DK, Nguyen DB, Malak R, Leroux JM, Carmant L, Saint-Hilaire JM, et al. Revisiting the role of the insula in refractory partial epilepsy. Epilepsia 2009; 50: 510-520.

46. Alomar S, Mullin JP, Smithason S, Gonzalez-Martinez J. Indications, technique, and safety profile of insular stereoelectroencephalography electrode implantation in medically intractable epilepsy. J Neurosurg 2018; 128: 1147-1157.
47. Guillaume J, Mazars G. Technique de resection de l'insula dans les epilepsies insulaires. Rev Neurol (Paris) 1949; 81: 900-903.

48. Silfvenius H, Gloor P, Rasmussen T. Evaluation of insular ablation in surgical treatment of temporal lobe epilepsy. Epilepsia 1964; 5: 307-320.

49. Yaşargil MG, von Ammon K, Cavazos E, Doczi T, Reeves JD, Roth P. Tumours of the limbic and paralimbic systems. Acta Neurochir 1992; 118: 40-52.

50. Yasargil MG, Krisht AF, Türe U, Al-Mefty O, Yasargil DCH. Microsurgery of insular gliomas: part II: opening of the sylvian fissure. Contemp Neurosurg 2002; 24: 1-5.

51. von Lehe M, Wellmer J, Urbach H, Schramm J, Elger CE, Clusmann H. Insular lesionectomy for refractory epilepsy: management and outcome. Brain 2009; 132: 1048-1056.

52. Malak R, Bouthillier A, Carmant L, Cossette P, Giard N, SaintHilaire JM, et al. Microsurgery of epileptic foci in the insular region: clinical article. J Neurosurg 2009; 110: 1153-1163.

\section{Clinical Practice Guidelines}

Clinical Practice Guidelines must include a short abstract. There should be an Introduction section addressing the objective in producing the guideline, what the guideline is about and who will benefit from the guideline. It should describe the population, conditions, health care setting and clinical management/diagnostic test. Authors should adequately describe the methods used to collect and analyze evidence, recommendations and validation. If it is adapted, authors should include the source, how, and why it is adapted? The guidelines should include not more than 50 references, 2-4 illustrations/tables, and an algorithm. 